\title{
Search for Ultralight Scalar Dark Matter from Pulsar Timing
}

\author{
Konstantin Postnov ${ }^{1}$ and Nataliya Porayko ${ }^{2}$ \\ ${ }^{1}$ M.V. Lomonosov Moscow State University, Faculty of Physics, \\ and Sternberg Astronomical Institute \\ email: pk@sai.msu.ru \\ ${ }^{2}$ M.V. Lomonosov Moscow State University, Faculty of Physics, \\ and Max Planck Institute for Radio Astronomy, Bonn \\ email: porayko.nataliya@gmail.com
}

\begin{abstract}
We perform a Bayesian analysis of pulsar-timing residuals from the NANOGrav pulsar-timing array to search for a specific form of stochastic narrow-band signal produced by oscillating gravitational potential (Gravitational Potential Background) in the Galactic halo. Such oscillations arise in models of warm dark matter composed of an ultralight massive scalar field $\left(m \simeq 10^{-23} \mathrm{eV}\right.$ ). The propagation of an electromagnetic signal from a pulsar through the time-dependent spacetime will leave an imprint in the pulsar timing, much like a gravitational wave. From the physical point of view, this is the classical Sachs-Wolfe effect. A distinctive feature of the pulsar-timing residuals due to GBP produced by a variable scalar field is that the amplitude of the TOA residuals should be independent of the pulsar location in the sky. In the monochromatic approximation, the stringent upper limit (95\% C.L.) on the variable gravitational potential amplitude is found to be $\Psi_{c}<1.14 \times 10^{-15}$, corresponding to the characteristic strain $h_{c}=2 \sqrt{3} \Psi_{c}<4 \times 10^{-15}$ at $f=1.75 \times 10^{-8} \mathrm{~Hz}$. In the narrow-band approximation, the upper limit of this background energy density is $\Omega_{G P B}<1.27 \times 10^{-9}$ at $f=1.75 \times 10^{-8} \mathrm{~Hz}$. These limits are an order of magnitude higher than the expected signal amplitude assuming all Galactic dark matter is made of such scalar particles. The applied analysis of the pulsar-timing residuals can be used to search for any narrow-band stochastic signals with different correlation properties. As a by-product, parameters of the red noise present in four NANOGrav pulsars were found.
\end{abstract}

Keywords. pulsar timing, ultralight scalar field, Bayesian approach

\section{Introduction}

Pulsar timing arrays (PTAs) are used to detect GWs in the low-frequency (nHz) range (Sazhin 1978; Detweiler 1979; Foster \& Backer 1990). Here we use PTA data to search for a Gravitational Potential Background (GPB) formed by hypothetical ultralight scalar field in the halo of our Galaxy, which can be a viable warm dark matter candidate (Khmelnitsky \& Rubakov 2014). The oscillating pressure of this field induces variations of gravitational potentials with $\mathrm{nHz}$ frequencies which could be probed with PTA technique in a similar way as traditional GWs.

\section{Pulsar-timing response and method of data analysis}

The pulses from pulsars moving in time-dependent gravitational potentials $\Phi$ and $\Psi$ are shifted due to the Sachs-Wolfe effect. Due to small virial velocity $v$ in our Galaxy, the frequency broadening of the signal $\Delta f / f=(v / c)^{2} \simeq 10^{-6}$ is much lower than frequency resolution of PTAs. Thus, the signal can be treated as a monochromatic line 


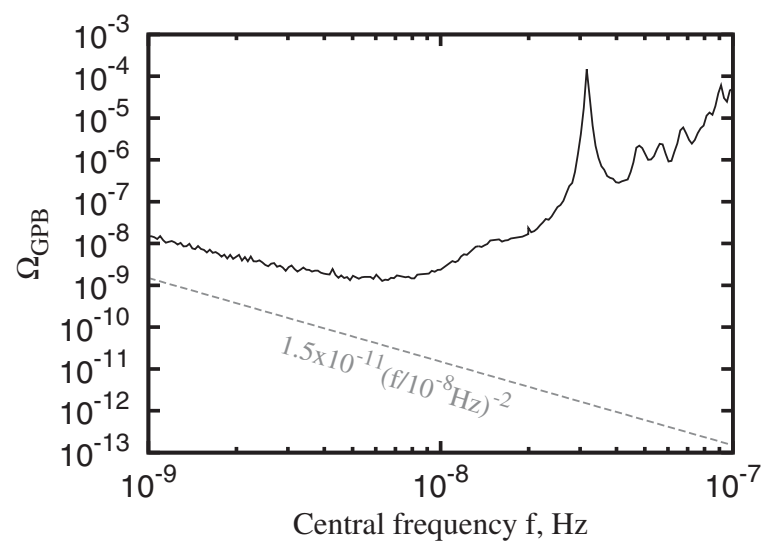

Figure 1. Upper limit on the amplitude of variable GPB $\Psi_{c}$ as a function of the central frequency $f$. Shown is the case of the narrow-band approximation (black line) for the $95 \%$ confidence level.

or a stochastic narrow-band noise. In the first case the form of the residuals is

$$
R(t)=\frac{\Psi_{c}}{2 \pi f}\left\{\left(\sin \left(2 \pi f t+2 \alpha\left(\mathbf{x}_{e}\right)-\sin \left(2 \pi f(t-D / c)+2 \alpha\left(\mathbf{x}_{p}\right)\right)\right\},\right.\right.
$$

where $D$ is the distance to the pulsar, $\alpha\left(\mathbf{x}_{e}\right), \alpha\left(\mathbf{x}_{p}\right)$ are the field phase on Earth and at the pulsar, respectively, $\Psi_{c}$ is the variable potential amplitude to be constrained from PTA timing analysis. In the narrow-band approximation, the expected signal is stochastic with power contained within the frequency band $\delta f$ around the central frequency $f$. The corresponding covariance matrix is:

$$
C_{\mathrm{GPB}}\left(\tau_{i j}\right)=\zeta_{\alpha \beta} \frac{\Psi_{c}^{2} \delta f}{\pi^{2} f^{3}} \cos \left(f \tau_{i j}\right) .
$$

In both cases we have taken into account the red intrinsic pulsar noise. The resultant covariance matrix has the form: $C=C_{\mathrm{WN}}+C_{\mathrm{RN}}+C_{\mathrm{GPB}}$.

In contrast to GWB, the angular correlation coefficient $\zeta_{\alpha \beta}=1 / 2\left(1+\delta_{\alpha \beta}\right)$ for GPB is monopole and does not depend on angular separations between pulsars which makes GPB similar to clock errors. However, broad-band red-colored nature (Tiburzi et al. 2015) makes it possible to distinguish clock errors from the considered GPB.

The Bayesian approach (van Haasteren \& Levin 2013) was applied to real NANOGrav dataset (Demorest et al. 2013) from 12 pulsars. The results are shown in Figure 1.

The best upper limit is $\Omega_{\mathrm{GPB}}<1.27 \times 10^{-9}$ at $f=6.2 \times 10^{-9} \mathrm{~Hz}$, which corresponds to $\Psi_{c}<1.5 \times 10^{-15}$, an order of magnitude higher than the predicted value (Porayko \& Postnov 2014). To obtain more stringent limits, pulsars with low red noise component and longer observational timespan should be used.

\section{Acknowledgement}

The work is supported by RSF grant 14-12-00203.

\section{References}

Demorest, P. B., Ferdman, R. D., Gonzalez, M. E., et al. 2013, ApJ, 762, 94

Detweiler, S. 1979, ApJ, 234, 1100

Foster, R. S. \& Backer, D. C. 1990, ApJ, 361, 300

Khmelnitsky, A. \& Rubakov, V. 2014, J. Cosmol. Astropart. Phys., 2, 019 
Porayko, N. \& Postnov, K., 2014, Phys. Rev. D., 90, 062008

Sazhin, M. V. 1978, SvA, 22, 36

Tiburzi, C., Hobbs, G., Kerr, M., et al. 2015, MNRAS, in press; arXiv:1510.02363

van Haasteren, R. \& Levin, Y. 2013, MNRAS, 428, 1147 